\title{
REDUCED EXPRESSIONS IN SEMIDIRECT PRODUCTS OF COXETER GROUPS
}

\author{
TOM EDGAR ${ }^{1}$
}

\begin{abstract}
Any normal reflection subgroup $\widetilde{W}$ of a Coxeter system $(W, S)$ is a factor in a semidirect product decomposition of $W$ as described by Bonnafé and Dyer. Namely, $S$ is the union of two subsets $I$ and $J$ such that no element of $I$ is conjugate to an element of $J, \widetilde{W}$ is the subgroup generated by $W_{I^{-}}$ conjugates of elements of $J$, and $W$ is the semidirect product of $W_{I}$ by $\widetilde{W}$. This note describes the reduced expressions of elements of the form $w x w^{-1}$ with $w \in W_{I}$ and $x \in W_{J}$ in terms of reduced expressions of $x$ and a suitable element of $W_{I}$.
\end{abstract}

प

\section{INTRODUCTION AND PRELIMINARIES}

Following Bonnafé and Dyer, we consider a Coxeter system $(W, S)$ in its internal semidirect product decomposition with respect to two subsets $I, J \subset S$ such that $I \cap J=\emptyset$ and $I \cup J=S$ and with the requirement that no element of $I$ is conjugate to an element of $J$. The latter statement says that the vertex set of the Coxeter graph of $(W, S)$ can be divided into two subsets with only edges with even label connecting the two. Let $T$ represent the set of reflections of $W$. In [4] (see also [1]), it is shown that $W$ is the semidirect product of $W_{I}$ and the normal subgroup $\widetilde{W}$ generated by $W_{I}$-conjugates of elements of $J$. We observe that any normal reflection subgroup $\widetilde{W}$ of $W$ arises from this type of decomposition of $S$. Then, we describe the reduced expressions of elements of the form $w x w^{-1}$ where $w \in W_{I}$ and $x \in W_{J}$ in terms of reduced expressions of $w$ and $x$. From this, we get a slightly stronger result describing reduced expressions of reflections of the form $w t w^{-1}$ with $t \in W_{J} \cap T$ and $w \in W_{I}$. For general results about Coxeter systems consult [5].

\section{Statement of Main Result}

We begin with the same setup as [1. We let $(W, S)$ be a Coxeter system with $\ell: W \rightarrow \mathbb{N}$ the corresponding length function. Furthermore, assume that $S=I \cup J$ with $I \cap J=\emptyset$ and that no element of $I$ is conjugate to an element of $J$. We denote by $W_{I}$ and $W_{J}$ the standard parabolic subgroups generated by $I$ and $J$ respectively. For any $K, L \subset S$, we let $W^{K}:=W / W_{K}$ (respectively ${ }^{L} W:=W_{L} \backslash W$ ) be the set of shortest right coset representatives of $W_{K}$ in $W$ (respectively the set of shortest left coset representatives of $W_{L}$ in $W$ ). Then we define ${ }^{L} W^{K}:={ }^{L} W \cap W^{K}$ to be the set of shortest representatives for the double cosets $W_{L} \backslash W / W_{K}$ in $W$. For brevity, we will use the following notation $W_{K}^{L}:=W_{K} \cap W^{L}$. Additionally, let $T=\cup_{w \in W} w S w^{-1}$ be the set of reflections of $W$. If $\mathcal{P}(T)$ denotes the power set of

Date: February 20, 2009.

${ }^{1}$ Department of Mathematics, University of Notre Dame, Notre Dame, IN 46556; tedgar@nd.edu 
$T$, then we let $N: W \rightarrow \mathcal{P}(T)$ be given by $N(w)=\{t \in T \mid \ell(t w)<\ell(w)\}$. We define a new set $\widetilde{J}=\left\{w s w^{-1} \mid w \in W_{I} ; s \in J\right\} \subset T$, and then let $\widetilde{W}$ be the subgroup of $W$ generated by $\widetilde{J}$. Part a) of the following result is due to [4, Proposition 2.1]; part b), along with a stronger version of part a), is proven (independently) in [1.

Theorem 1.1 (Gal; Bonnafé, Dyer). Let $(\widetilde{W}, \widetilde{J})$ be as above.

a) $(\widetilde{W}, \widetilde{J})$ is a Coxeter system and $W=\widetilde{W} \rtimes W_{I}$.

b) Every element $w \in W_{I}$ is the unique element of the coset $w \widetilde{W}$ with minimal length.

We complement [1] by noting that any normal reflection subgroup of a Coxeter group appears as the normal component of a semidirect product decomposition for $W$ as in Theorem 1.1 .

Proposition 1.2. Let $W^{\prime}$ be any normal reflection subgroup of $W$. Then, there exists a subset $I \subset S$ such that $W=W_{I} \ltimes W^{\prime}$, that is, any normal reflection subgroup of $W$ is a normal semidirect factor of $W$.

Proof. Let $W^{\prime \prime}$ be any reflection subgroup of $W$ and let $W^{\prime}$ be its normal closure in $W$. Then, define $J \subset S$ to be $J=S \cap\left(\bigcup_{w \in W} w\left(W^{\prime \prime} \cap T\right) w^{-1}\right)$. Since $W^{\prime}$ is normal and generated by the reflections it contains, we have that $W^{\prime}$ is generated by the $W$-conjugates of $J$. Now, we let $I=S \backslash J$, and let $\widetilde{J}=\left\{w s w^{-1} \mid w \in W_{I} ; s \in J\right\}$ and $\widetilde{W}$ be the group generated by $\widetilde{J}$. By Theorem 1.1 , we know that $\widetilde{W}$ is normal; since $J \subset \widetilde{W}$, we have $w J w^{-1} \subset \widetilde{W}$ for all $w$ and so $W^{\prime} \leq \widetilde{W}$. Thus, $W^{\prime}=\widetilde{W}$. Also by the theorem, we know that $W=W_{I} \ltimes \widetilde{W}$ so $W=W_{I} \ltimes W^{\prime}$.

So any normal reflection subgroup arises from a decomposition of $S$ into two disjoint subsets such that no element in one set is conjugate to an element of the other set.

Now, we can move on to our main result. For any element $x \in W$, define the set $x^{\perp}:=\{s \in S \mid s r=r s$ for all $r$ appearing in a reduced expression for $x\}$,

which is well defined since any reduced expression for $x$ uses the same set of simple reflections.

Now, for fixed $w \in W_{I}$, elements of the form $w x w^{-1}$ with $x \in W_{J}$ form a standard parabolic subgroup of $\widetilde{W}$, namely the subgroup generated by the set

$$
J^{\prime}=\left\{w s w^{-1} \mid s \in J\right\} \subset \widetilde{J} .
$$

In Lemma 2.4 we will show that every $W_{I^{-}}$-conjugate of an element in $W_{J}$ is uniquely expressible as $w x w^{-1}$ with $w \in W_{I}^{I \cap x^{\perp}}$ and $x \in W_{J}$. Our goal is to understand how reduced expressions of elements of this form reflect the semidirect product decomposition of $W$.

For any expression $r_{1} \ldots r_{n}$ with $r_{i} \in S$ for all $i$ and $D \subset\{1, \ldots, n\}$, we denote by $\prod_{D} r_{i}$ the product $r_{i_{1}} \ldots r_{i_{j}}$ where $i_{1}<i_{2}<\cdots<i_{j}$ and $D=\left\{i_{1}, \ldots, i_{j}\right\}$. The main result here characterizes the reduced expressions of elements of the form $w x w^{-1}$ where $w \in W_{I}$ and $x \in W_{J}$.

Theorem 1.3. Suppose that $S=I \cup J, I \cap J=\emptyset$, and no element of $I$ is conjugate to an element of $J$, and define $(\widetilde{W}, \widetilde{J})$ as above. Suppose that $x \in W_{J}$ and $w \in$ $W_{I}^{I \cap x^{\perp}}$ with $\ell(x)=m$. Fix a reduced expression $w x w^{-1}=r_{1} \ldots r_{d}$ for $w x w^{-1}$. From 
this expression, we can define two sets $A_{J}:=\left\{i \mid r_{i} \in J\right\}$ and $A_{I}:=\left\{i \mid r_{i} \in I\right\}$. Then the following are true:

(1) $\left|A_{J}\right|=m$.

(2) $\prod_{A_{J}} r_{i}$ is a reduced expression of $x$.

(3) There exists $B \subset A_{I}$ where $\prod_{B} r_{i}$ is a reduced expression of $w$ and $\prod_{A_{I} \backslash B} r_{i}$ is a reduced expression of $w^{-1}$.

(4) Let $i \in A_{J}$, so $r_{i} \in J$. Then

(a) $r_{i} r_{j}=r_{j} r_{i}$ for all $j \in B$ with $j>i$.

(b) $r_{i} r_{k}=r_{k} r_{i}$ for all $k \in A_{I} \backslash B$ with $k<i$.

(5) Let $i \in B$ and $j \in A_{I} \backslash B$ with $j<i$. Then $r_{i} r_{j}=r_{j} r_{i}$.

(6) $N\left(w x w^{-1}\right) \cap \widetilde{W}=w N(x) w^{-1}$.

This theorem basically says that every reduced expression for $w x w^{-1}$ is essentially of the form: (reduced expression of $w$ )(reduced expression of $x$ )(reduced expression of $w^{-1}$ ) up to commutation relations. Following this, we can say a bit more for the case of $x$ being a reflection $x=t \in W_{J} \cap T$.

Corollary 1.4. Consider the same setup as Theorem 1.3. Let $w \in W_{I}^{I \cap x^{\perp}}$ and $x=t \in W_{J} \cap T$ with $\ell(t)=2 k+1$ so that wtw $^{-1}=r_{1} \ldots r_{e+1} \ldots r_{2 e+1}$ is a reduced expression. Then following the notation from Theorem 1.3. the following are true:

(1) $e+1 \in A_{J}$ and $\left|\left\{i \in A_{J} \mid i<e+1\right\}\right|=k$.

(2) $B=\left\{i \in A_{I} \mid i<e+1\right\}$ and so $A_{I} \backslash B=\left\{i \in A_{I} \mid i>e+1\right\}$.

\section{Proof of Theorem 1.3}

To prove the main result, we need two auxiliary results. To get to these results, we first remind the reader of the following standard results regarding shortest double coset representatives.

Proposition 2.1. Let $K, L \subset S$. The following hold:

(1) For $w \in{ }^{K} W^{L}, W_{K} \cap w W_{L} w^{-1}=W_{K \cap w L w^{-1}}$.

(2) Let $w \in{ }^{K} W^{L}$, then any $x \in W_{K} w W_{L}$ has a unique expression $x=u w v$ with $u \in W_{K}^{K \cap w L w^{-1}}$ and $v \in W_{L}$. Furthermore, for this expression, $\ell(u w v)=\ell(u)+\ell(w)+\ell(v)$.

Proof. See 2, 2.7]. The proofs given are for finite Coxeter groups, but the proofs easily extend to any Coxeter group.

This proposition is the main ingredient for the proofs of Lemma 2.3 and Lemma 2.4 below. To prove these lemmas, it is also useful to have the following:

Lemma 2.2. Let $I, J \subset S, I \cap J=\emptyset$, and let $x \in W_{J}$. Then $x \in{ }^{I} W^{I}$ and $I \cap x I x^{-1}=I \cap x^{\perp}$.

Proof. Clearly multiplying $x$ by a simple reflection from $I$ on the left or right must increase the length so $x \in{ }^{I} W^{I}$. Now the fact that $I \cap x I x^{-1} \subset I \cap x^{\perp}$ follows since if $r=x s x^{-1}$ for some $r, s \in I$, then $r x=x s$. Now, both $r x$ and $x s$ are reduced expressions (for any reduced expression of $x$ ) and since all reduced expressions use the same simple reflections we must have $r=s$ since $x \in W_{J}$. Thus we have $r \in x^{\perp}$. Containment in the other direction is trivial. 
Lemma 2.3. Let $I, J \subset S$ with $I \cap J=\emptyset$. Let $x \in W_{J}$, and let $w \in W_{I}^{I \cap x^{\perp}}$ (that is, $w$ is in $W_{I}$ and is a shortest right coset representative of $\left.W_{I \cap x^{\perp}}\right)$. Then $\ell\left(w x w^{-1}\right)=2 \ell(w)+\ell(x)$.

Proof. This is just a combination of Proposition 2.1 and Lemma 2.2

The next lemma, which follows from Proposition 2.1. gives a normal form for

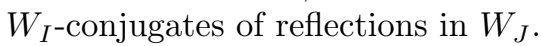

Lemma 2.4. Suppose that $S=I \cup J$ and $I \cap J=\emptyset$. Every $W_{I}$-conjugate of an element in $W_{J}, w x w^{-1}\left(w \in W_{I}, x \in W_{J}\right)$ is uniquely expressible as uxu ${ }^{-1}$ with $u \in W_{I}^{I \cap x^{\perp}}$ and $x \in W_{J}$.

Proof. Since $x \in{ }^{I} W^{I}$ then by Proposition 2.1 part (2), we know that $w x w^{-1}=u x v$ with $u \in W_{I}^{I \cap x^{\perp}}$ (by Lemma 2.2 and $v \in W_{I}$. We note that $w=u^{\prime} w^{\prime}$ where $u^{\prime} \in W_{I}^{I \cap x^{\perp}}$ and $w^{\prime} \in W_{I \cap x^{\perp}}$ so that $w x w^{-1}=u^{\prime} w^{\prime} x w^{-1}=u^{\prime} x w^{\prime} w^{-1}=u^{\prime} x u^{\prime-1}$ so that by necessity $u^{\prime}=u$ and so $v=u^{-1}$ in the unique decomposition of $w x w^{-1}$. Thus, $w x w^{-1}=u x u^{-1}$ with $u \in W_{I}^{I \cap x^{\perp}}$.

Remark 2.5. Lemma 2.4 can also be interpreted in the following way. Let $x, x^{\prime} \in W_{J}$ and $w, w^{\prime} \in W_{I}$ then $w x w^{-1}=w^{\prime} x^{\prime} w^{\prime-1}$ if and only if $x=x^{\prime}$ and $w^{-1} w^{\prime} \in W_{I \cap x^{\perp}}$.

From this point on, we will assume that $S=I \cup J$ with $I \cap J=\emptyset$ and no element of $I$ is conjugate to an element of $J$.

Remark 2.6. Before we move the proof of Theorem 1.3 notice that since $\widetilde{W} \triangleleft$ $W$, then for $w$ and $x$ as in Theorem 1.3 with $w x w^{-1}=r_{1} \ldots r_{d}$ we have $t_{i}:=$ $r_{1} \ldots r_{i-1} r_{i} r_{i-1} \ldots r_{1} \in \widetilde{W}$ if and only if $r_{i} \in J$.

Proof of Theorem 1.3. Suppose that $w=u_{1} \ldots u_{n}$ and $x=s_{1} \ldots s_{m}$ are reduced expressions for $w$ and $x$ respectively. Then, we know by Lemma 2.3 that $w x w^{-1}=$ $u_{1} \ldots u_{n} s_{1} \ldots s_{m} u_{n} \ldots u_{1}$ is a reduced expression for $w x w^{-1}$. Therefore, according to Remark 2.6. $\left|N\left(w x w^{-1}\right) \cap \widetilde{W}\right|=\ell(x)$. Using the remark in reverse, if $w x w^{-1}=$ $r_{1} \ldots r_{2 n+m}$ is any reduced expression, there must be $i_{1}<i_{2}<\cdots<i_{k}$ such that $r_{i_{j}} \in J$ for all $j$ and $k=\ell(x)$. Then $A_{J}=\left\{i_{1}, \ldots, i_{\ell(x)}\right\}$, and this proves (1).

Now, any reduced expression can be achieved by applying braid operations to a given reduced expression. We already have a particular reduced expression, namely $w x w^{-1}=u_{1} \ldots u_{n} s_{1} \ldots s_{m} u_{n} \ldots u_{1}$, so $A_{J}=\{n+1, \ldots, n+m\}, A_{I}=\{1, \ldots, n, n+$ $m+1, \ldots, 2 n+m\}$ and $B=\{1, \ldots, n\}$. Thus (2)-(5) clearly hold for this reduced expression. Now we proceed to prove (2)-(5) by induction on the number of braid operations needed to change from $u_{1} \ldots u_{n} s_{1} \ldots s_{m} u_{n} \ldots u_{1}$.

Suppose $w x w^{-1}=r_{1} \ldots r_{2 n+m}$ is a reduced expression satisfying (2)-(5) of the theorem. For this expression we have $A_{I}, A_{J}$ and $B$ defined. Clearly, braid operations involving simple reflections, $r_{i}$, with indices, $i$, that are both in $A_{J}$, both in $B$, or both in $A_{I} \backslash B$ are just rearranging $x, w$, or $w^{-1}$ respectively and are thus giving new reduced expressions for $x, w$, or $w^{-1}$ respectively; consequently, the theorem holds. This leaves us with three cases.

Case 1: $r_{i} \in I$ and $r_{i+1} \in J$ (or vice versa). If $r_{i}$ and $r_{i+1}$ do not commute, then we know that $r_{i-1}=r_{i+1} \in J$ or $r_{i+3}=r_{i+1} \in J$ by the restriction that no element of $I$ is conjugate to an element of $J$. However, either of these situations contradicts the fact that $\prod_{A_{J}} r_{i}=r_{i_{1}} \ldots r_{i-1} r_{i+1} r_{i+3} \ldots r_{i_{\ell(x)}}$ is a reduced expression of $x$, which holds because $w x w^{-1}=r_{1} \ldots r_{2 n+m}$ is 
a reduced expression satisfying (2). Therefore, $r_{i}$ commutes with $r_{i+1}$ and thus $w x w^{-1}=r_{1} \ldots r_{i+1} r_{i} \ldots r_{2 n+m}$ is a reduced expression satisfying (2)-(5). Case 2: $i \in B$ and $i+1 \in A_{I} \backslash B$ so $r_{i} \in I$ and $r_{i+1} \in I$. Let $r:=r_{i}$ and $s:=r_{i+1}$ and suppose that these simple reflections do not commute. Then to perform a braid operation, either $r_{i-1}=s$ or $r_{i+2}=r$ (or both). Without loss of generality, we assume $r_{i+2}=r$. We note that in this case we must have $i+2 \in A_{I} \backslash B$ otherwise we contradict the fact that $\prod_{B} r_{i}$ is a reduced expression for $w$. However, by induction, we know that $r=r_{i}$ commutes with all $r_{j}$ with $j<i$ and $j \in A_{J}$ and simultaneously, $r=r_{i+2}$ commutes with all $r_{j}$ with $j>i+2$ and $j \in A_{J}$. This implies that $r$ commutes with all $r_{j}$ with $j \in A_{J}$ so that $r$ commutes with $x$. Finally, we see also by induction that $r=r_{i+2}$ commutes with all $r_{k}$ with $k \in B$ and $k>i$. Thus, $w$ has a reduced expression ending in $r$ and $r$ commutes with $x$ contradicting the fact that $w \in W_{I}^{I \cap x^{\perp}}$. So, $r_{i}$ must commute with $r_{i+1}$ and $w x w^{-1}=r_{1} \ldots r_{i} r_{i+1} r_{i+2} \ldots r_{2 n+m}$ is a new reduced expression satisfying (2)-(5).

Case 3: $i \in A \backslash B$ and $i+1 \in B$ so $r_{i} \in I$ and $r_{i+1} \in I$. Since the reduced expression we are currently in satisfies the theorem by induction, property (5) implies that $r_{i}$ and $r_{i+1}$ commute.

So we have shown that any braid operation that can occur must either be a commutation relation or be a rearrangement of $w, x$, or $w^{-1}$ separately.

Lastly, we prove part (6). As we have seen, $\left|N\left(w x w^{-1}\right) \cap \widetilde{W}\right|=\ell(x)=|N(x)|$. Clearly, $w N(x) w^{-1} \subset \widetilde{W}$ since $x \in W_{J}, W_{J} \subset \widetilde{W}$ and $\widetilde{W}$ is normal. So let $t \in$ $N(x)$, that is $\ell(t x)<\ell(x)$. Since $\ell\left(w x w^{-1}\right)=2 \ell(w)+\ell(x)$, then $\ell\left(w t w^{-1} w x w\right)=$ $\ell\left(w t x w^{-1}\right)<\ell\left(w x w^{-1}\right)$ because $t x$ has smaller length than $x$. Thus, $w t w^{-1} \in$ $N\left(w x w^{-1}\right)$ for all $t \in N(x)$, and so $w N(x) w^{-1} \subset N\left(w x w^{-1}\right)$. Finally, we have seen that $w N(x) w^{-1} \subset N\left(w x w^{-1}\right) \cap \widetilde{W}$ and these sets are both finite with the same cardinality so they must be equal.

Lastly, the corollary follows from the following information about reflections. For a reflection $t$, we say that $t=r_{1} r_{2} \ldots . r_{n}$ is a palindromic reduced expression if $r_{i}=r_{n-i+1}$ for all $i \leq \frac{n-1}{2}$. The following lemma is due to [3].

Lemma 2.7. If $t=r_{1} \ldots r_{2 m+1}$ is a reduced expression for $t \in T$, then $t=$ $r_{1} \ldots r_{m} r_{m+1} r_{m} \ldots r_{1}$ is a reduced palindromic expression of $t$.

Corollary 2.8. Suppose $I$ and $J$ are disjoint, $S=I \cup J$ and no element of $I$ is conjugate to any element of $J$. Let $w \in W_{I}$ and $t \in W_{J} \cap T$. Now, for any reduced expression $w t w^{-1}=r_{1} \ldots r_{n+1} \ldots r_{2 n+1}$ then we have $w t w^{-1}=r_{1} \ldots r_{n} r_{n+1} r_{n} \ldots r_{1}$ and so $r_{n+1} \in J$.

Proof. This is just a restatement of Lemma 2.7 along with the fact that $w t w^{-1} \in \widetilde{W}$ so that $w t w^{-1}$ must be conjugate to an element of $J$.

Proof of Corollary 1.4. Let $w t w^{-1}=r_{1} \ldots r_{e+1} \ldots r_{2 e+1}$. The proof follows directly from Corollary 2.8, which tells us that $e+1 \in A_{J}$. Thus, we can never have a braid operation occur between the middle element and a simple reflection in $I$. Therefore, we can never have $i \in B$ and $j \in A_{I} \backslash B$ with $j<i$ since that would have required a braid relation between $r_{i}$ and the middle element or $r_{j}$ and the middle element, which would in turn have given us a reduced expression for $w t w^{-1}$ with $r_{e+1} \in I$. 


\section{REFERENCES}

[1] C. Bonnafé and M.J. Dyer. Semidirect product decomposition of Coxeter groups. preprint (2008), available at arXiv:0805.4100

[2] Roger W. Carter. Finite groups of Lie type. Pure and Applied Mathematics (New York). John Wiley \& Sons Inc., New York, 1985. Conjugacy classes and complex characters, A WileyInterscience Publication.

[3] Matthew Dyer. Reflection subgroups of Coxeter systems. J. Algebra, 135(1):57-73, 1990.

[4] Światosław R. Gal. On normal subgroups of Coxeter groups generated by standard parabolic subgroups. Geom. Dedicata, 115:65-78, 2005.

[5] James E. Humphreys. Reflection groups and Coxeter groups, volume 29 of Cambridge Studies in Advanced Mathematics. Cambridge University Press, Cambridge, 1990.

Department of Mathematics, University of Notre Dame, Notre Dame, IN 46556

E-mail address: tedgar@nd.edu 